\title{
Memoria del pasado reciente en la escuela: saberes prácticos de profesores y mediaciones multimodales
}

\author{
Juan Carlos Amador-Baquiro ${ }^{1} \bullet$ \\ Diego Fernando Barragán-Giraldo ${ }^{2} \odot$ \\ Cristina Álvarez-Vargas ${ }^{3} \odot$
}

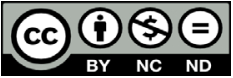

1. Universidad Distrital Francisco José de Caldas, Colombia.

jcamadorb@udistrital.edu.co

2. Universidad de La Salle, Colombia.

3. Fundación Cinde, Colombia.

Recibido: $26 / 05 / 2021$

Revisado: 10/06/2021

Aprobado: 27/10/2021

Publicado: 01/01/2022

\section{Resumen}

La pedagogía de la memoria del pasado reciente es un campo epistémico cuyo objetivo es que los estudiantes lean críticamente los hechos de violencia sociopolítica ocurridos en determinadas coordenadas de tiempo y espacio, problematizados por distintos actores sociales desde el presente. Así, en este artículo se analizan el saber práctico de un grupo de docentes de colegios oficiales de Bogotá y las mediaciones multimodales que ellos implementan para la formación en memoria del pasado reciente. En un diseño cualitativo de tres fases, se evidencia cómo los profesores, desde su saber práctico, privilegian objetivos de formación relacionados con los efectos del conflicto en las víctimas, con la exclusión y la desigualdad y con la ciudadanía memorial. Asimismo, ellos implementan tres tipos de mediaciones para el desarrollo de estos objetivos de formación con sus estudiantes: la integración de lo gestual, lo corporal, lo alfabético y lo visual; la producción de piezas audiovisuales; y la articulación de lenguajes análogos y digitales, a partir de la creación de proyectos de tipo multimedial e hipermedial.

Palabras clave: memoria, pasado reciente, saber práctico, mediaciones, multimodalidad

Para citar este artículo: Amador-Baquiro, J. C., Barragán-Giraldo, D. F., \& Álvarez-Vargas, C. (2022). Memoria del pasado reciente en la escuela: saberes prácticos de profesores y mediaciones multimodales. Praxis \& Saber, 13(32), e12911. https:/doi.org/10.19053/22160159.v13.n32.2022.12911 


\title{
Memory of the recent time at school: teachers' practical knowledge and multimodal mediations
}

\begin{abstract}
The pedagogy of the memory of recent time is an epistemic field whose objective is to enable students to critically read the events of socio-political violence that occurred in particular coordinates of time and space, problematized by different social actors at the present time. Thus, this article analyzes the practical knowledge of a group of teachers from official schools in Bogotá and the multimodal mediations that they use in teaching the memory of the recent time. In a three-phase qualitative design, it was found how teachers, from their practical knowledge, privilege learning objectives related to the effects of the conflict on victims, to exclusion and inequality, and to a memorial citizenship. They also implement three types of mediations to develop these learning objectives with their students: the integration of gestural, corporal, alphabetic and visual elements; the production of audiovisual material; and the articulation of analog and digital languages through the creation of multimedia and hypermedia projects.
\end{abstract}

Keywords: : memory, recent time, practical knowledge, mediations, multimodality

\section{Memória do passado recente na escola: os saberes práticos dos professores e as mediações multimodais}

\section{Resumo}

A pedagogia da memória do passado recente é um campo epistêmico cujo objetivo é que os estudantes leiam criticamente os fatos da violência sociopolítica que ocorreram em certas coordenadas de tempo e espaço, problematizados por diferentes atores sociais no presente. Assim, este artigo analisa o saber prático de um grupo de professores de escolas oficiais em Bogotá e as mediações multimodais que eles implementam para a formação em memória do passado recente. Num desenho qualitativo trifásico, é evidente como os professores, a partir de seus saberes práticos, privilegiam objetivos de aprendizagem relacionados com os efeitos do conflito sobre as vítimas, com a exclusão e a desigualdade e com a cidadania memorial. Ainda, eles implementam três tipos de mediação para o desenvolvimento destes objetivos de aprendizagem com seus alunos: a integração de elementos gestuais, corporais, alfabéticos e visuais; a produção de produtos audiovisuais; e a articulação de linguagens analógicas e digitais, baseada na criação de projetos multimídia e hipermídia.

Palavras-chave: memória, passado recente, saber prático, mediações, multimodalidade 
${ }^{1}$ La pedagogía de la memoria es un campo epistémico y de reflexión que promueve la construcción de subjetividades políticas, afectadas directa o indirectamente por contextos de violencia sociopolítica (Herrera \& Merchán, 2012; Ortega et al., 2014), como contribución a la valoración del otro en su diferencia, en su adversidad y en su capacidad de agencia (Murillo, 2017). Asimismo, propende por la formación de actores sociales en torno a la interpretación crítica de las experiencias límite del pasado reciente ${ }^{2}$ que han configurado la vulneración sistemática de derechos humanos, en el marco de violencias relacionadas con guerras civiles, conflictos armados, dictaduras, genocidios y otros hechos atroces. Por esta razón, también busca contribuir a la formación de agentes sociales capaces de efectuar valoraciones éticas y políticas de las posiciones de diversos sectores de la sociedad sobre estos hechos, entre ellos, integrantes del Estado, actores del conflicto, organizaciones sociales y organizaciones de víctimas, quienes, desde distintas motivaciones, convierten estos acontecimientos del pasado en problemas actuales (Kriger, 2011; Rubio, 2010).

En los ámbitos nacional e internacional se distinguen tres tipos de debates académicos:

1. la enseñanza del pasado reciente.

2. la construcción de currículos para la pedagogía de la memoria.

3. la función de las narrativas en el trabajo pedagógico sobre el pasado reciente.

En relación con el primer aspecto, sobresalen: debates en torno a los criterios de los docentes para seleccionar los contenidos que orientarán el análisis de hechos de violencia sociopolítica en la escuela; las contradicciones que estos pueden llegar a evidenciar frente a versiones oficiales; y una serie de didácticas que buscan incentivar el pensamiento crítico a partir del trabajo con actores sociales implicados (Arias, 2016; Carretero \& Borrelli, 2008; Domínguez, 2015; Kriger, 2011; Levín, 2007).

En lo concerniente a lo curricular, surgen estudios que examinan las tendencias curriculares de algunos países frente a la enseñanza del pasado reciente e investigaciones que evidencian tanto la coexistencia de relatos hegemónicos, institucionales y subalternos sobre el pasado reciente en los currículos escolares como la presencia de orientaciones ideológicas en los referentes curriculares del Estado que simplifican o niegan ciertos acontecimientos en sintonía con los gobiernos de turno (Jiménez et al., 2012; Rodríguez \& Sánchez, 2009; Rubio, 2010).

En cuanto a los aportes de las narrativas a estos objetivos de formación, se destacan perspectivas que analizan las relaciones entre memoria, pedagogía y narrativa, las funciones performativas de la narrativa en la construcción de la memoria colectiva y social y la implementación de narrativas multimodales en la configuración de prácticas educativas conducentes a la transformación de sistemas de significación sobre el pasado reciente (Amador, 2021; Herrera \& Pertuz, 2016; Mèlich, 2006; Murillo, 2017).

1 El presente artículo de investigación se inscribe en el proyecto de investigación Pedagogías de la memoria para la reparación simbólica de niños y niñas en colegios oficiales de Bogotá: un enfoque desde la multimodalidad, financiado por el Centro de Investigaciones y Desarrollo Científico de la Universidad Distrital Francisco José de Caldas (Código 4-160-602-19) con el apoyo de la Universidad de la Salle.

2 La historia reciente-también llamada pasado cercano, pasado reciente, historia contemporánea e historia actual, entre otras hace referencia a eventos que se consideran traumáticos y que se convierten objeto de debate ético y político en las sociedades desde el presente. 
En el caso de Colombia, existe una serie de referentes curriculares orientados por el Estado relacionados con este campo, los cuales, desde hace cerca de dos décadas, han pretendido establecer los contenidos que los docentes deben impartir en la escuela para cumplir con estas demandas sociales, políticas y pedagógicas. Dentro de dichos referentes se destacan los Estándares de competencias en el área de Ciencias Sociales (Ministerio de Educación Nacional [MEN], 2004), los Estándares de competencias ciudadanas (MEN, 2003) y la Cátedra de Paz (Ley 1732, Congreso de Colombia, 2014). En relación con este último referente, por medio del Decreto 1038 (Presidencia de la República de Colombia, 2015), se puntualizaron algunos contenidos que involucran temas relacionados con la memoria del pasado reciente: justicia y derechos humanos, uso sostenible de recursos naturales, protección de las riquezas culturales y naturales de la nación, resolución pacífica de conflictos, prevención del acoso escolar, diversidad y pluralidad, participación política, memoria histórica, dilemas morales, proyectos de impacto social, historia de los acuerdos de paz nacionales e internacionales, proyectos de vida y prevención de riesgos.

No obstante, además de incluir la memoria histórica como un tema adicional dentro de un largo listado de contenidos, sin hilo conductor alguno, la cátedra es apenas un conjunto de enunciados prescriptivos que carece de orientaciones pedagógicas y didácticas para el logro de los propósitos ampliamente argumentados por investigadores en los campos de la enseñanza del pasado reciente y la pedagogía de la memoria. Por otro lado, es importante señalar que en la actualidad la construcción de propuestas pedagógicas, especialmente relacionadas con este campo epistémico y de reflexión, debe atender al menos a dos aspectos fundamentales:

1. la experiencia y el saber práctico de los profesores.

2. la configuración de mediaciones multimodales que, desde diversas narrativas y modos semióticos, contribuyan a leer críticamente los hechos del pasado reciente y le permitan al estudiante aportar iniciativas para la restauración moral de las víctimas y participar en la construcción de proyectos sociales hacia el futuro.

Como se pudo observar, estas dos dimensiones del campo de la pedagogía de la memoria no han sido profundizadas por otros investigadores.

En relación con la primera dimensión, se puede señalar que la experiencia es un proceso humano y social ampliamente relacionado con el saber práctico, comprendido como un elemento constitutivo de la condición humana que posibilita la inserción del ser humano en el mundo (Barragán, 2015; Flyvbjerg, 2001). Este tipo de saber hace posible el despliegue de capacidades que se ponen en juego en la medida que las personas actúan según situaciones particulares (Ricoeur, 2010). De este modo, la experiencia hace referencia al acumulado de prácticas que, al ser implementadas en el marco de la temporalidad existencial de los actores sociales, se asumen como propias, lo cual configura situaciones de los individuos experimentados (Gadamer, 1975). Esta perspectiva, que articula saber práctico, temporalidad y acción, contribuye a la configuración de memorias sociales, colectivas y públicas, entendidas como dispositivos de tipo ético, político y pedagógico que permiten leer críticamente las experiencias límite pretéritas con el fin de construir nuevos porvenires desde el presente (Carretero \& Borrelli, 2008; Ortega et al., 2014; Rubio, 2020). En este caso, los profesores 
pueden hacer contribuciones fundamentales, desde su experiencia y su saber práctico, para el cumplimiento de estos propósitos.

Por su parte, las mediaciones multimodales se refieren a situaciones de aprendizaje basadas en el uso y en la apropiación de contenidos constituidos de múltiples modos semióticos y medios educativos - análogos y/o digitales-, los cuales, además de optimizar prácticas de interacción y de trabajo colaborativo, aportan a la transformación de los sistemas de significación, a la construcción de posiciones sobre hechos sociales y a la generación de proyectos alternativos (Amador, 2020; González, 2018). Además de asumir que los seres humanos producen significados de acuerdo con su contexto situacional y con los recursos materiales y simbólicos disponibles (Kress, 2010), este enfoque propone dos procesos fundamentales en la formación escolar:

1. propiciar prácticas educativas, basadas en la integración de modos semióticos de tipo gestual, kinestésico, verbal, alfanumérico, visual, sonoro y/o audiovisual, con el fin de representar los conocimientos escolares por medio de herramientas de modelización (Jewitt, 2008; Mangui et al., 2016).

2. promover formas de comunicación bidireccional mediadas, en las cuales los estudiantes se alimentan comunicacional e informativamente todos con todos (Aparici \& Silva, 2012; Amador, 2018).

El trabajo con este tipo de mediaciones puede contribuir ampliamente al enriquecimiento de los aprendizajes y de los sistemas de significación de los estudiantes sobre la memoria del pasado reciente (Arias, 2016; Centro Nacional de Memoria Histórica [CNMH], 2018).

De acuerdo con lo expuesto, el presente artículo tiene como propósito presentar los resultados de una investigación que pretendió analizar experiencias y saberes prácticos de docentes situados en instituciones educativas de Bogotá, así como la gestión pedagógica que estos llevan a cabo para el diseño e implementación colaborativa de mediaciones multimodales sobre la memoria del pasado reciente. Si bien estos profesores conocen los referentes curriculares mencionados, sus iniciativas no se basan únicamente en estas prescripciones orientadas por el Estado, sino también en: sus propias experiencias de vida; las condiciones sociales, políticas y culturales del contexto local y nacional; sus posiciones frente al conflicto armado; sus miradas frente a la política educativa; y los sistemas de intereses, necesidades y expectativas de sus estudiantes.

\section{Metodología}

El enfoque cualitativo desde el cual se desarrolló este trabajo y la profundidad de la experiencia de los actores que participaron en este son el principal derrotero de esta investigación (Creswell, 1997; Galeano, 2009). Si bien no se trata de una investigación que emplee una muestra representativa con el fin de dar cuenta de la generalidad, la riqueza de experiencias de los participantes seleccionados evidencia que estos se pueden convertir en casos emblemáticos y referentes significativos (Creswell \& Miller, 2000) para otros docentes, para otras prácticas y para otras apuestas por la memoria del pasado reciente en la escuela. En 
esta dirección, la investigación asumió como punto de partida que los profesores son sujetos activos en la transformación de las realidades que experimentan.

Por otro lado, aunque algunos estudios sobre experiencias y saberes prácticos de docentes sugieren trabajar desde la etnografía educativa con énfasis en la observación de campo, desde aquello que los actores sociales dicen y dicen que hacen en el contexto del aula, en este estudio se privilegió el diálogo y la reflexión sistemática con cada uno de estos con el fin de comprender la capacidad de agencia de sus prácticas educativas. Además de reflexionar colectivamente sobre la posibilidad de potenciar y/o transformar dichas prácticas (Díaz-Bazo, 2017; Kemmis et al., 2014), el estudio buscó fomentar escenarios de experimentación pedagógica para coconstruir mediaciones conducentes a la formación de subjetividades políticas en los estudiantes que contribuyan a la lectura crítica de los hechos del pasado reciente y a la problematización de los puntos de vista que distintos actores producen sobre estos acontecimientos con arreglo a determinados intereses. A partir de estas consideraciones, se trazaron y se diseñaron las siguientes fases de investigación:

\section{Identificación y alistamiento}

Se realizó un balance respecto a estudiantes víctimas del conflicto armado en Bogotá, en educación prescolar, básica y media en instituciones oficiales. Según la Alcaldía Mayor de Bogotá (2019), a julio de 2018, 68588 niños, niñas y jóvenes en esta situación estaban matriculados en las instituciones educativas públicas de la ciudad. En el informe mencionado se identificaron las localidades con mayor concentración de estudiantes víctimas: Ciudad Bolívar -11 955-, Bosa - 10 767-, Kennedy - 10 269- y Usme -7006-, para un total de 39997 estudiantes. Luego, en estas zonas se localizaron experiencias significativas de maestros que tuvieran en sus aulas estudiantes víctimas y que desarrollaran acciones en relación con las pedagogías de la memoria. Así, se ubicaron diez instituciones educativas, con 3678 estudiantes víctimas del conflicto armado (figura 1).

Luego se eligieron experiencias docentes en relación con el concepto de muestra significativa, intencional, donde "cada unidad - o conjunto de unidades - es cuidadosa e intencionalmente seleccionada por sus posibilidades de ofrecer información profunda y detallada sobre el asunto de interés para la investigación" (Martínez, 2012, p. 614). De este modo, se ubicó una experiencia por institución, liderada por uno o varios profesores. Los criterios de inclusión para la selección fueron:

- haber desarrollado iniciativas con alto contenido de agencia sobre memoria del pasado reciente.

- ser profesor en una institución pública de Bogotá, en alguna de las localidades mencionadas.

- tener disposición para participar en el proceso de formación en mediación multimodal.

De los diez profesores seleccionados, tres contaban con 20 años o más de experiencia profesional; seis, entre 10 y 19 años; y una, menos de 5.

\section{Figura 1}


Instituciones educativas participantes en la investigación. Mapa de Bogotá por localidades.

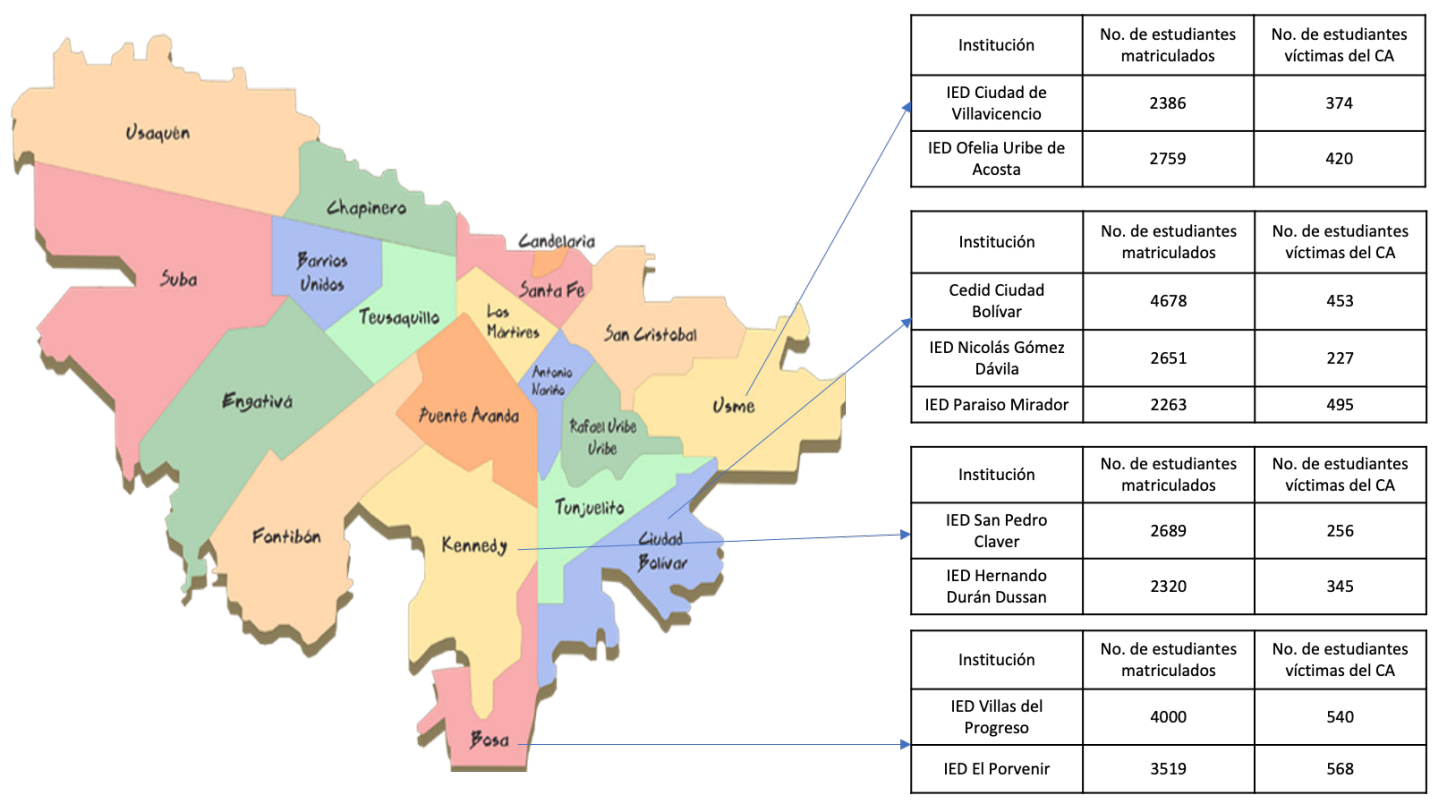

\section{Caracterización y análisis}

Se recolectó la información a través de visitas y observaciones preliminares a las instituciones educativas. No obstante, la base del corpus fueron las entrevistas semiestructuradas dirigidas a los profesores que lideraban las experiencias. Estas entrevistas se desarrollaron con el fin de profundizar en el saber práctico experiencial. El análisis de la información se asumió desde el análisis de contenido (Huberman \& Miles, 1991), en el cual se interpretan las dinámicas, las disposiciones y la estructura de la comunicación, así como los contextos en que se producen las prácticas. Estas acciones se desarrollaron desde los dos ejes centrales del estudio. El primer eje experiencia y saber práctico- se desglosó a través de las categorías emergentes: conocimiento de sí, conocimiento del contenido del pasado reciente, conocimiento del estudiante, y posición frente a la política pública. En relación con el eje de mediaciones multimodales, las categorías emergentes fueron: propósitos de las mediaciones; y modos semióticos y medios utilizados.

\section{Formación multimodal}

Una vez analizada la información, con los docentes participantes se llevó a cabo un proceso de formación en el que reflexionaron sobre sus prácticas y realizaron ajustes a estas. Los temas práctico-teóricos trabajados fueron:

- Pedagogía de emociones para la paz, mediaciones: narrativas y fotomemorias.

- Memoria histórica y otras narrativas.

- Mediaciones: Cine, literatura, teatro.

- Reparación Simbólica.

- Mediaciones: comunicación/arte, experiencias estéticas. 
- Enfoque diferencial en los procesos de pedagogías de la memoria.

Como producto final, los docentes presentaron sus iniciativas pedagógicas de memoria en lógica de producción multimodal — análoga o digital—. Así mismo, se realizaron nuevas entrevistas para recoger impresiones sobre sus experiencias y el ajuste a las mismas.

En esta ruta, diseñada a la medida de este trabajo, se cuidó que estuviese presente la transferibilidad de los resultados, situación que se refiere a la capacidad de aplicar los hallazgos a otros contextos similares "a partir de la descripción rica y profunda de cada fenómeno en su contexto y no tiene como fundamento el número de casos estudiados" (Martínez, 2012, p. 615). De esta forma, se valora el ejercicio de agencia que las experiencias producen, lo cual se ajusta a los lineamientos de la investigación cualitativa. Por último, respecto a los criterios éticos de la investigación, se realizaron los consentimientos informados y la anonimización correspondiente de los archivos. También se asumieron los principios de trabajo investigativo con víctimas de conflicto y posconflicto (Carvallo, 2019; Mckenzie, 2019), especialmente por la particularidad política del contexto colombiano.

\section{Resultados}

En esta sección se presentarán los resultados de los dos ejes comprensivos de trabajo. El primero remite a la experiencia y el saber práctico de los profesores. El segundo considera las mediaciones multimodales para la memoria del pasado reciente en el contexto escolar.

\section{Saber práctico y experiencias de profesores}

Conocimiento de sí. Esta dimensión hace referencia a la capacidad de narrar la experiencia de sí (Carr, 2004; Foucault, 1988) a partir de procesos de autoobservación, autorreflexión y autodominio (Herrera \& Martínez, 2018), como posibilidad ontológica y epistémica para tramitar el presente y los planes hacia el futuro. En tal sentido, se exploraron aspectos de la biografía del profesor y su trayectoria profesional como posibilidad de indagación sobre su valor pedagógico, personal, ético y político (Antunes, 2011; Severino, 2011), con énfasis en sus vivencias frente al conflicto armado y frente a los procesos de paz y reconciliación. Al respecto, se encontraron tres tipos de resultados:

1. experiencias directas con el conflicto armado.

2. trayectorias de trabajo comunitario y militante.

3. la docencia como posibilidad de transformación, desde abajo.

En relación con el primer resultado, se encontró que tres profesores han experimentado a lo largo de sus vidas situaciones propias o cercanas al conflicto armado, asociadas con el desplazamiento forzado y la toma de poblaciones. Dos de ellos manifestaron haber tenido relación con estos hechos desde su infancia:

- Profesora 9 (P9): Trabajé en Putumayo cinco años. Fui perseguida... por personas de estas comunidades que pertenecían a la guerrilla y al paramilitarismo.

- P4: Creo que mi vida ha estado muy marcada por la guerra... llegamos desplazados a Bogotá. 
- P6: Soy del Caquetá, fuimos desplazados por la violencia, tuvimos que migrar a pueblos pequeños para que no nos encontraran.

En cuanto a las trayectorias de trabajo comunitario y militante, se encontró que tres profesores cuentan con experiencias de voluntariado en poblaciones vulnerables y militancia con comunidades de base, las cuales se desarrollaron en ámbitos rurales y urbanos. Ejercían este tipo de actividad antes de dedicarse a la docencia o al vincularse a ella:

- P7: Llegué a Colombia y estudié Teología... Tuve discrepancias y me uní al movimiento Torrevieja de Teología de la Liberación. Eso me hizo conocer de cerca la situación de violencia y pobreza de los campesinos.

- P3: Me enrolé en el trabajo con grupos juveniles en Ciudad Bolívar, desde mi labor docente.

- P6: Inicié el trabajo de memoria histórica con jóvenes de barrios populares.

En relación con el ejercicio de la docencia como posibilidad de transformación, desde abajo, dos profesores plantearon que su oficio es una opción política para la transformación social y que su función principal es subvertir las estructuras de reproducción social por medio de prácticas educativas significativas y críticas:

- P10: A mí no me gusta la sociedad como está y creo que una de las cosas para cambiarla, o una de las herramientas más apropiadas para cambiar esto, es la educación. Por eso decidí ejercer la docencia.

- P2: Aprendí a reconocer que tenía ciertas facultades o habilidades para poder desempeñar esta labor e incidir en la transformación de la sociedad.

Conocimiento del contenido enseñable sobre el pasado reciente. El conocimiento del contenido que se enseña implica considerar las intenciones formativas, en este caso, para producir aprendizajes y significados en torno a la lectura crítica del conflicto armado y/o la violencia sociopolítica en el país. Así, es importante tener en cuenta que la enseñanza del pasado reciente no tiene una larga trayectoria en los planes de estudio en Colombia en comparación con áreas del conocimiento de amplia tradición en el currículo escolar (Arias, 2016; Arias \& Herrera, 2018), situación que puede explicar la heterogeneidad en las respuestas de los profesores. Al respecto, estas se sintetizaron en tres perspectivas:

1. la importancia del estudio del pasado reciente en los planes de estudio.

2. la incorporación de contenidos sobre género, diversidad y diferencia como aportes a la construcción de paz.

3. el trabajo en la escuela con la memoria histórica como alternativa para la formación de una ciudadanía memorial.

En relación con la primera perspectiva, dos profesores mencionaron que una fundamentación histórica para los estudiantes que adopte con mirada crítica los hechos de la violencia sociopolítica vividos en los últimos cincuenta años es un aporte valioso para construir una visión de transformación en el presente: 
- P2: Optamos por hacer un cambio en el plan de estudios, enfocado en desarrollar ejercicios de memoria histórica... para acercarlos un poco más a la historia de la violencia política del país.

- P10: La historia del pasado reciente no se estudia para admirar qué pasó, sino que debe servir como insumo para transformar las condiciones de dominación.

Sobre la segunda perspectiva, dos profesores afirman que el tratamiento de temas sobre el conflicto armado y la paz en la escuela requiere el abordaje de asuntos atinentes a la exclusión y la desigualdad por situaciones relacionadas con el género, la sexualidad, la etnia y la clase social:

- P9: En las clases hacemos ejercicios para reconocer lo que nos pasó en la guerra, pero también para que los estudiantes construyan posturas políticas sobre problemáticas de violencia de género.

- P3: Me inclino siempre por reconocer esos elementos del conflicto y que los estudiantes sean capaces de reconocerse a sí mismos y se valoren desde su identidad étnica y de género.

En cuanto a la tercera perspectiva, dos profesores plantean que los trabajos en memoria histórica contribuyen a desarrollar conocimientos, habilidades y valores de carácter éticopolítico, fundamentales para que los estudiantes realicen lecturas críticas de la realidad y tomen decisiones de índole personal, familiar y comunitario, en la perspectiva de un modelo de ciudadanía memorial ${ }^{3}$ :

P10: La construcción ciudadana es lograr que la persona incida en su entorno y decida sobre su vida, sobre la comunidad y sobre el mundo.

P3: El trabajo en memoria histórica aporta a la formación política de los estudiantes... ellos pueden reconocerse como parte de una historia, de una comunidad política y de un sistema que no solo debe reproducir sino transformar.

Conocimiento del estudiante. Este aspecto aborda los conocimientos de los profesores en torno a los intereses, las necesidades y las expectativas de sus estudiantes. De acuerdo con Amade-Escot y O'Sullivan (2007), este tipo de conocimiento adquiere mayor importancia cuando el profesor hace explícitas las necesidades de acogida que requieren sus estudiantes para incorporarse a las dinámicas de aprendizaje y de participación en la escuela. Este aspecto también busca reconocer si los profesores tienen en cuenta no sololas problemáticas y situaciones de vulnerabilidad de sus estudiantes, sino también sus potencialidades y capacidades. Luego de la sistematización de datos, se encontraron tres posiciones:

1. estudiantes como individuos con carencias, afectados por el conflicto armado y necesitados de apoyo.

2. estudiantes como sujetos de saber y experiencia.

3. estudiantes comprendidos como sujetos políticos.

3 La construcción de subjetividades políticas, desde una pedagogía de la memoria, contribuye a la generación de iniciativas ciudadanas que visibilicen las formas de afectación a las víctimas y sobrevivientes, y propongan alternativas para la restauración moral de estas, desde respuestas específicas de los ofensores, el Estado y otros actores sociales, pero también desde los testimonios y experiencias de estas. A este proceso Jelin (2002) le denomina ciudadanía memorial. 
En relación con la primera, dos profesores plantean que sus estudiantes han sido afectados por el conflicto armado y que requieren apoyo dada su situación de vulnerabilidad:

- P7: Muchos de mis estudiantes son hijos directos de desplazados, desmovilizados del paramilitarismo y reinsertados de las guerrillas.

- P2: Para nosotros fue una sorpresa saber que teníamos más de 400 estudiantes víctimas del conflicto armado en nuestra escuela.

Por otro lado, un profesor asocia esta situación con problemáticas como la baja autoestima y la dificultad para resolver conflictos en la vida cotidiana escolar:

- P9: Generalmente tienen baja autoestima y dificultades para relacionarse con sus compañeros. Por esta razón, decidimos trabajar la memoria histórica.

Sobre el carácter de sujetos de saber, dadas las experiencias de los estudiantes en escenarios como la familia, el barrio y la comunidad, un profesor resalta las potencialidades de sus estudiantes para abordar temas de memoria histórica y procesos de paz en la escuela:

- P6: Iba en el transporte público y vi cantar rap a un joven que pedía plata... Me di cuenta de que era mi estudiante... Le dije que utilizara su talento para contar historias de su realidad y fue una de las mejores experiencias de mi vida profesional.

Otro educador resalta que sus estudiantes son sujetos políticos y que su prioridad está en formarlos para que lideren proyectos sobre memoria histórica:

- P5: Creo que mis estudiantes son sujetos políticos... La idea es que conozcan lo que nos pasó y lideren proyectos para transformar la cultura de violencia que ha afectado a varias generaciones.

Posición frente a la política educativa. Este aspecto aborda los conocimientos y las valoraciones de los profesores sobre la política educativa, así como las implicaciones de estas posiciones en la formación de los estudiantes. El aspecto también indaga los conocimientos y las valoraciones del profesor frente a las orientaciones curriculares establecidas por el Estado en materia de educación para la paz, historia del pasado reciente y memoria histórica. Al respecto, se identificaron dos perspectivas en los profesores:

1. críticas de las políticas educativas que han impulsado, desde inicios del 2000, la estandarización del currículo, las pruebas censales y la burocratización de la escuela.

2. críticas de las orientaciones de la Cátedra de Paz, la cual, desde sus miradas, es confusa en materia curricular y pedagógica.

En relación con el primer aspecto, cinco profesores señalan que varias medidas impuestas por el Estado - entre ellas, la implementación de estándares de competencias, la aplicación de pruebas censales y la incorporación de procedimientos empresariales en la escuelaresponden a compromisos con organismos internacionales, como la OCDE, y que estas no atienden a las necesidades de aprendizaje de los estudiantes:

- P5: Lo que busca el Gobierno es que los estudiantes presenten pruebas estandarizadas, no que se formen como sujetos críticos. 
- P10: Nos han dicho estándares... pero estos enfoques desconocen lo que pasa en nuestra realidad colombiana.

- P4: La escuela se convirtió en una burbuja de contenidos y disciplinas... dispuesta para el mercado, debido a las condiciones que impone la OCDE.

- P1: Es preocupante la cantidad de formatos que debemos llenar y los trámites que debemos hacer para que evalúen nuestro desempeño.

En relación con el segundo resultado, algunos profesores afirman que, aunque vienen implementando la Cátedra de Paz, es necesario que el Estado dé orientaciones curriculares y pedagógicas sobre este saber escolar, especialmente si se tiene en cuenta la alta presencia de estudiantes víctimas del conflicto armado en el sistema educativo público:

- P9: Es necesario contar con herramientas pedagógicas para trabajar en el aula con los estudiantes víctimas del conflicto, pero también con los hijos de los desmovilizados.

- P3: La Cátedra de Paz solo ofrece temas y contenidos... necesitamos construir didácticas con TIC para trabajar con estudiantes víctimas y no víctimas.

\section{Mediaciones multimodales}

Propósitos de las mediaciones. Los profesores participantes han implementado mediaciones multimodales sobre memoria del pasado reciente, apoyados tanto en modos semióticos diversos como en medios análogos y digitales. En estas mediaciones aparecen intenciones formativas sobre los objetivos orientados hacia la adquisición de conocimientos, valores y habilidades para que los estudiantes se sensibilicen frente a los hechos atroces de la violencia política, participen en la construcción de la memoria histórica y se imaginen horizontes de futuro posibles:

- P3: Que sean capaces de reflexionar sobre lo que nos ha pasado como sociedad.

- P7: Las actividades buscan que los estudiantes sean sensibles ante la realidad para no repetir situaciones de violencia y construir sus proyectos de vida.

Por otro lado, algunos docentes buscan propiciar en sus estudiantes el liderazgo de proyectos escolares y/o comunitarios que contribuyan a construir paz, a partir de estrategias relacionadas con la solución de conflictos, con el respeto a las diferencias y con la reconciliación:

- P6: La idea es que propongan proyectos para apoyar a su comunidad, pues muchos de sus integrantes son desplazados por la guerra.

- P5: Que aprendan a resolver los conflictos de manera creativa.

- P9: Que construyan posiciones frente a la discriminación por género o etnia.

Modos semióticos y medios utilizados. Las mediaciones multimodales empleadas por los profesores se caracterizan por su variabilidad didáctica y comunicativa. En general, se puede decir que los profesores utilizan distintos modos semióticos de acuerdo con los objetivos de formación y con la disponibilidad de medios educativos en el centro escolar. Al respecto, se identificó que los profesores implementan tres maneras distintas de combinación o ensamblaje 
de modos semióticos (Amador, 2020; Mangui et al., 2016):

1. integración de la gestualidad, la corporalidad, el texto alfabético y el texto visual, por medio de actividades lúdicas.

2. combinación de modos semióticos de tipo verbal, alfabético, visual y sonoro, a través de la interpretación y producción de piezas audiovisuales.

3. la articulación de lenguajes análogos y digitales por medio de la creación de proyectos de tipo multimedial e hipermedial.

En relación con la primera forma de integración semiótica, se observa que los profesores emplean medios convencionales, basados en el lenguaje alfabético, como los libros de texto, la prensa y los informes del CNMH, con el fin de fomentar en los estudiantes la interpretación de información relacionada con hechos del pasado reciente. Luego, a partir de estos ejercicios, proponen prácticas lúdicas como juegos de mesa, cartografías y murales, que privilegian lenguajes de tipo gestual, corporal y kinestésico:

- P1: Después de revisar los hechos victimizantes del informe ¡Basta ya!, hicimos un juego de mesa con el mapa de Colombia.

- P4: Partí de los temas de la Cátedra de Paz y propusimos una cartografía del cuerpo... Luego hicimos una jornada de muralismo.

- P2: Utilizamos la cartilla sobre la masacre de El Salado y luego trabajamos en una actividad llamada El tendero de la memoria.

En cuanto al segundo tipo de articulación semiótica, se encuentra que algunos profesores buscan que sus estudiantes transiten de la interpretación de información alfabética a la producción de contenidos sobre el pasado reciente, por medio de la producción de piezas visuales y audiovisuales. Algunos profesores mencionan que, antes de realizar procedimientos técnicos, los estudiantes deben hacer consultas sobre los hechos seleccionados, interactuar con las víctimas y producir narrativas que plasmen, a modo de huella, testimonio o ficción, las situaciones de la guerra o las iniciativas de paz:

- P4: Utilizo mucho material publicado en YouTube. Esto los inspira para que realicen sus propias producciones.

- P5: Les propuse preguntas como: “¿Cuál es la importancia de la memoria histórica? ¿Cómo reparar a las víctimas?”. Luego realizaron su propio escrito e hicieron un proyecto fotográfico.

- P7: Hemos traído víctimas del conflicto al colegio para que cuenten su testimonio... Eso los sensibilizó y les dio elementos para hacer fotografías que representaron el conflicto armado.

- La tercera forma de combinación semiótica se caracteriza por prácticas colectivas y conectivas, conducentes a la producción de proyectos multimediales e hipermediales. Además de integrar modos semióticos de tipo verbal, corporal, alfabético y audiovisual, estas mediaciones suelen apoyarse tanto en medios análogos como digitales, con el fin 
de fomentar en los estudiantes conocimientos y habilidades para la implementación de proyectos sobre memoria histórica. Estos proyectos se apoyan en recursos digitales como redes sociales, aplicaciones, softwares de edición y herramientas de realidad aumentada:

- P3: Usamos literatura colombiana sobre el conflicto. Los estudiantes entrevistaron víctimas y diseñaron un portal web con sus historias de vida.

- P8: Aprendieron a trabajar crónicas sobre el conflicto armado y el proceso de paz entre el Gobierno y las Farc. Posteriormente, utilizaron una aplicación de realidad aumentada para ubicar imágenes de desaparecidos en una galería de la memoria. Las personas debían utilizar una aplicación de celular para leer códigos QR y ver sus rostros.

\section{Conclusiones}

En relación con la experiencia y con el saber práctico del profesor, se observa que las historias de vida influidas por situaciones del conflicto armado, así como las experiencias de trabajo comunitario y militante, tienen una relación directa con la valoración que varios profesores hacen sobre el estudio del pasado reciente y con el trabajo pedagógico en memoria histórica en la escuela. Mientras que los profesores que vivieron hechos de violencia política - desde su niñez o en su ejercicio docente- evidencian altos grados de sensibilidad y convicción frente al tema dada su condición de víctimas del conflicto, aquellos que han tenido experiencias con comunidades afectadas por la violencia muestran su compromiso, empatía y solidaridad por la situación de desventaja o adversidad de los otros. A esto se añade la posición de algunos profesores que explicitan su deber moral de entender el dolor de los demás, especialmente cuando estos han transitado por experiencias límite.

En consecuencia, al haber vivido de diversas maneras el conflicto armado, la desigualdad y la exclusión, estos docentes son profesores experimentados para la pedagogía de la memoria del pasado reciente, dado que sus experiencias personales, familiares y comunitarias les han permitido relacionarse consigo mismos, desde la reflexividad, y con los demás, desde la otredad y la alteridad. En esta línea de reflexión, también es importante tener en cuenta a aquellos docentes que proponen el ejercicio de la docencia como alternativa para transformar la realidad y no para reproducir el orden social y cultural (Gilje \& Erstad, 2017; Giroux, 2015). En tal sentido, se observa que los profesores experimentados, además de sentir compasión por los demás, asumen que su labor es un compromiso político que implica formar sujetos no solo para que aprendan determinados contenidos, sino también para transformar estructuras opresivas en calidad de agentes sociales (Miño, 2020).

Por otro lado, los resultados muestran que existe una alta heterogeneidad de enfoques y perspectivas sobre los objetivos de formación y los contenidos por desarrollar con los estudiantes. $\mathrm{Al}$ respecto, llama la atención que el estudio del pasado reciente alude no solo al estudio de hechos de violencia sociopolítica, sino también al compromiso de profesores y estudiantes de resignificar ese pasado desde el presente y de examinar problemáticas actuales relacionadas con la exclusión y la desigualdad, a propósito de las herencias patriarcales, machistas, clasistas y adultocéntricas que ha dejado la guerra en las nuevas generaciones (Jaramillo et al., 2019). En tal sentido, aunque aún resulta difusa la secuencialidad y gradualidad de los contenidos 
de la memoria del pasado reciente en la escuela y su incorporación en los planes de estudios, es importante destacar el interés de la mayoría de los docentes por promover espacios de reflexión y de acción en torno a las diferencias (García, 2020) desde apuestas formativas por la construcción de una ciudadanía memorial (Jelin, 2002; Murillo, 2017).

En relación con el conocimiento del estudiante, los resultados evidencian que el $100 \%$ de los docentes participantes ha tenido altos grados de interacción, de diálogo y de cercanía con sus estudiantes. Mientras que unos profesores resaltan la situación de vulnerabilidad de sus estudiantes, dado que son víctimas evidentes del conflicto armado, otros destacan sus potencialidades como sujetos de saber, y otros los asumen como sujetos políticos con capacidades para participar, deliberar y decidir. Estos resultados llaman la atención dado que, al asumir a sus estudiantes como individuos con problemáticas debido a la exclusión y a la desigualdad de la que son objeto, con frecuencia los profesores terminan ejerciendo prácticas de estigmatización hacia ellos (McLaren, 2005). En este estudio se evidencia que la condición de víctimas no convierte a los estudiantes en sujetos deficitarios o anormales. Antes bien, al asumirlos como sujetos de saber y con capacidad de decisión, los profesores evidencian que otorgan un valor especial a sus experiencias fuera de la escuela (Gilje \& Erstad, 2017; Giroux, 2015).

Por último, con respecto a las posiciones de los profesores frente a las políticas educativas, se evidencia que ellos cuestionan claramente sus orientaciones ideológicas y que rechazan los mecanismos de reproducción de la exclusión social que el Estado hace a través del sistema educativo. En el $100 \%$ de los profesores se observa una clara inconformidad con la política de estandarización del currículo y la evaluación, la cual, desde sus puntos de vista, lesiona los procesos de formación de los estudiantes y los ancla en una lógica de reproducción tanto del orden social como de las estructuras de poder. Por otro lado, los resultados también muestran que existe un vacío en la formación en educación para la paz y el estudio del pasado reciente en la escuela. La Cátedra de Paz, al parecer, es insuficiente para alcanzar estos objetivos. En consecuencia, creen en un proceso alternativo de formación en el contexto de las mutaciones socioculturales contemporáneas (Virkkunnen, 2006).

En relación con las mediaciones multimodales, como se observó en los resultados, es frecuente el uso de mediaciones, a partir de múltiples modos semióticos, con el fin de abordar temas relacionados con el estudio del pasado reciente, con la articulación entre identidad, comunidad y territorio y con las problemáticas de las diferencias. Mientras que algunos profesores le dan prioridad a la integración entre la gestualidad, la corporalidad, lo alfabético y lo visual, otros incluyen lenguajes visuales, sonoros y audiovisuales en este tipo de situaciones educativas. Por último, están aquellos profesores que integran lenguajes y medios, tanto análogos como digitales, para el desarrollo de proyectos de carácter multimedial e hipermedial.

De acuerdo con los resultados, se puede decir que la primera tipología es muy importante dada la interacción que fomenta por medio de las actividades lúdicas. Sin embargo, es una situación educativa que asume a los estudiantes preferiblemente como usuarios e intérpretes de la mediación, la cual está previamente diseñada por el profesor. La segunda tipología, además de propiciar la interacción del estudiante con el dispositivo pedagógico y entre ellos, hace 
posible que este produzca contenidos a partir de proyectos que integran lo verbal y lo alfabético con lo visual y lo audiovisual. Esto ayuda a que el estudiante no solo interprete información de manera eficiente, sino que produzca contenidos a partir de la integración entre los sensorial y lo cognitivo. La tercera tipología de mediación, la cual integra lo verbal y lo alfabético con medios educativos análogos y digitales, además de propiciar la participación del estudiante en la producción de contenidos, fomenta el trabajo colaborativo y la cocreación.

Por otro lado, en las correlaciones entre los dos ejes de análisis, se observa que los profesores que vivieron experiencias directas de conflicto armado y aquellos que tuvieron experiencias de trabajo comunitario están abiertos a experimentar propuestas especialmente relacionadas con la situación de las víctimas, con los procesos de reparación y con los mecanismos de no repetición. Los profesores interesados en propiciar la reflexión sobre las diferencias desde el género, la sexualidad y la etnia buscan desarrollar con sus estudiantes trabajos especialmente relacionados con lo teatral, con el cuerpo y con la performatividad. Por último, aquellos profesores que tuvieron experiencias comunitarias y de militancia, se inclinan por desarrollar mediaciones orientadas hacia el debate público, la formación de una ciudadanía memorial y la beligerancia.

\section{Referencias}

Alcaldía Mayor de Bogotá. (2019). Escuelas que narran y resignifican la memoria. Guía Pedagogías de la memoria histórica, la reconciliación y la reparación simbólica para la atención de estudiantes víctimas del conflicto armado interno. Secretaría de Educación del Distrito de Bogotá; Universidad Distrital Francisco José de Caldas.

Amade-Escot, C., \& O'Sullivan, M. (2007). Research on content in physical education: theoretical perspectives and current debates. Physical Education and Sport Pedagogy, 12(3), 185-204. https://doi. org/10.1080/17408980701610144

Amador, J. (2018). Educación interactiva a través de narrativas transmedia: posibilidades en la escuela. Magis, Revista Internacional de Investigación en Educación, 10(2), 77-94. https://doi.org/10.11144/ Javeriana.m10-21.eint

Amador, J. (2020). Alfabetización multimodal crítica: textos complejos, comunicación del conocimiento y proyectos alternativos. En J. Amador, S. Rojas, \& R. Solano (Eds.), Comunicación-educación en contextos de globalización, neoliberalismo y resistencia (pp. 79-104). Universidad Distrital.

Amador, J. (2021). Memorias de hechos atroces, emprendimientos artístico-estéticos y visualidad: un estado de la cuestión. Encuentros, 19(1), 40-62. https://doi.org/10.15665/encuen.v19i01.2307

Antunes, C. (2011). Quanto vale um professor? Reais ou imaginários, alguns imprescindíveis, outros nem tanto. Vozes.

Aparici, R., \& Silva, M. (2012). Pedagogía de la interactividad. Comunicar, 9(38), 51-58. https://doi. org/10.3916/C38-2012-02-05

Arias, D. (2016). La memoria y la enseñanza de la violencia política desde estrategias audiovisuales. Revista Colombiana de Educación, (71), 253-278. https://doi.org/10.17227/01203916.71rce253.278

Arias, D., \& Herrera, M. (2018). Currículo sobre la enseñanza del pasado reciente y la violencia política en Colombia. Revista Clío \& Asociados, (27), 18-29. 
Barragán, D. (2015). El saber práctico: phrónesis. Hermenéutica del quehacer del profesor. Universidad de La Salle.

Carr, W. (2004). Philosophy and Education. Journal of Philosophy of Education, 38(1), 56-73. https://www. scirp.org/(S(i43dyn45teexjx455qlt3d2q))/reference/referencespapers.aspx?referenceid=1824725

Carretero, M., \& Borrelli, M. (2008). La enseñanza de la historia reciente: propuestas para pensar históricamente. En M. Carretero, \& J. Castorina (Eds.), La construcción del conocimiento histórico. Enseñanza, narración e identidades (pp. 101-130). Paidós.

Carvallo, S. (2019). L'éthique de la recherche entre réglementation et réflexivité. Revue d'Anthropologie des Connaissances, 13(2), 299-326. https://doi.org/10.3917/rac.043.0299.

Centro Nacional de Memoria Histórica. (2018). Recorridos de la memoria histórica en la escuela. Aportes de maestras y maestros en Colombia. CNMH.

Congreso de Colombia. (2014, 1 de septiembre). Ley 1732 de 2014. Por la cual se establece la Cátedra de la Paz en todas las instituciones educativas del país. https://www.funcionpublica.gov.co/eva/ gestornormativo/norma_pdf.php?i=59313

Creswell, J. (1997). Qualitative inquiry and research design: choosing among five traditions. Sage Publications.

Creswell, J., \& Miller, D. (2000). Determining validity in qualitative inquiry. Theory into Practice, 39(3), 124-130. https://doi.org/10.1207/s15430421tip3903_2

Díaz-Bazo, C. (2017). La investigación-acción en la educación básica en Iberoamérica. Una revisión de la literatura. Magis, Revista Internacional de Investigación en Educación, 10(20), 159-182. https://doi. org/10.11144/Javeriana.m10-20.iaeb

Domínguez, J. (2015). La enseñanza de la historia reciente y transición política en Colombia: constantes fracturas y nuevos horizontes. Polisemia, (20), 57-72. https://revistas.uniminuto.edu/index.php/ $\mathrm{POLI} /$ article/view/1281

Flyvbjerg, B. (2001). Making social science matter: Why social inquiry fails and how it can succeed again. Cambridge University Press.

Foucault, M. (1998). El sujeto y el poder. Revista Mexicana de Sociología, 50(3), 3-20.

Gadamer, H. (1975). Wahrheit und Methode. Mohr.

Galeano, M. (2009). Estrategias de investigación social cualitativa. El giro de la mirada. La Carreta.

García, N. (2020). Educación, memoria histórica y escuela: contribuciones para un estado del arte. Revista Colombiana de Educación, 1(79), 135-170. https://doi.org/10.17227/rce.num79-8918

Gilje, Ø., \& Erstad, O. (2017). Authenticity, agency and enterprise education studying. International Journal of Educational Research, (84), 58-67. http://dx.doi.org/10.1016/j.ijer.2016.05.012

Giroux, H. (2015). Pedagogías disruptivas y el desafío de la justicia social bajo regímenes neoliberales. Revista Internacional de Educación para la Justicia Social, (42), 13-27. https://revistas.uam.es/riejs/ article/view/2368

González, J. (2018). El enfoque multimodal del proceso de alfabetización. Educação em Revista, (34), 1-28. https://doi.org/10.1590/0102-4698177266 
Herrera, J., \& Martínez, A. (2018). El saber pedagógico como saber práctico. Pedagogía y Saberes, (49), 9-26. https://doi.org/10.17227/pys.num49-8167

Herrera, M., \& Merchán, J. (2012). Pedagogía de la Memoria y enseñanza de la historia reciente. Universidad Pedagógica Nacional.

Herrera, M., \& Pertuz, C. (2016). Cuento para no olvidar. Aportes a la Cátedra de la Paz desde el estudio de la violencia política y la narrativa testimonial. En P. Ortega (Ed.), Bitácora para la Cátedra de la Paz. Formación de maestros y educadores para una Colombia en paz (pp. 187-218). Universidad Pedagógica Nacional.

Huberman, M., \& Miles, M. (1991). Analyse des donnés qualitatives. De Boeeck.

Jaramillo, J., Parrado, E., \& Louidor, W. (2019). Geografías violentadas y experiencias de reexistencia. El caso de Buenaventura, Colombia, 2005-2015. Íconos, (64), 111-136. http://dx.doi.org/10.17141/ iconos.64.2019.3707

Jelin, E. (2002). Los trabajos de la memoria. Siglo XXI.

Jewitt, C. (2008). Multimodal Classroom Research. Review of Research in Education, (32), 241-267.

Jiménez, A., Infante, R., \& Cortés, R. (2012). Escuela, memoria y conflicto en Colombia. Un ejercicio del estado del arte de la temática. Revista Colombiana de Educación, (62), 287-314. http://doi. org/10.17227/01203916.62rce\%p

Kemmis, S., Wilkinson, J., Edwards-Groves, C., Hardy, I., Peter, G., \& Bristol, L. (2014). Changing practices, changing education. Springer.

Kress, G. (2010). Multimodality: A social semiotic approach to contemporary communication. Routledge.

Kriger, M. (2011). La enseñanza de la historia reciente como herramienta clave de la educación política. Narrativas escolares y memorias sociales del pasado dictatorial argentino en las representaciones de jóvenes estudiantes de la Ciudad de Buenos Aires y conurbano (2010-2011). Persona y Sociedad, (3), 29-52.

Levín, F. (2007). El pasado reciente en la escuela, entre los dilemas de la historia y la memoria. En G. Schujman, \& I. Siede (Eds.), Ciudadanía para armar. Aportes para la formación ética y política (pp. 157-178). Aique.

Mangui, D., Lagos, P., \& Pizarro, D. (2016). Oportunidades de producción semiótica en el aula. Mirada sociocultural y multimodal. Literatura y Lingüistica, (34), 197-220. https://doi. org/10.29344/0717621X.34.1470

Martínez, C. (2012). El muestreo en investigación cualitativa. Principios básicos y algunas controversias. Ciência \& Saúde Coletiva, 7(13), 613-619. https://www.scielo.br/j/csc/a/ VgFnXGmqhGHNMBsv4h76tyg/?lang=es

Mckenzie, T. (2019). Ethnographier un projet de recherche Nord-Sud: Entre différences épistémologiques, enjeux éthiques et rapports de pouvoir. Revue d'Anthropologie des Connaissances, 13(2), 425-454. https://doi.org/10.3917/rac.043.0425

McLaren, P. (2005). La vida en las escuelas. Una introducción a la pedagogía crítica en los fundamentos de la educación. Siglo XXI.

Mèlich, J. (2006). El trabajo de la memoria o el testimonio como categoría didáctica. Enseñanza de las Ciencias Sociales, (5), 115-124. https://www.raco.cat/index.php/EnsenanzaCS/article/view/126322 
Ministerio de Educación Nacional de Colombia. (2003). Estándares de Competencias Ciudadanas. MEN.

Ministerio de Educación Nacional de Colombia. (2004). Estándares de competencias en el área de Ciencias Sociales. MEN.

Miño. R. (2020). Iniciativas para la igualdad y la transformación de la educación secundaria obligatoria en Cataluña. Posibilidades y tensiones. Izquierdas, (49), 2378-2404. https://dialnet.unirioja.es/ servlet/articulo?codigo $=7695041$

Murillo. G. (2017). Pedagogía de la memoria entre la guerra y la paz. Revista Educação em Questão, 55(44), 32-50. https://bibliotecadigital.udea.edu.co/handle/10495/15815

Ortega, P., Merchán, J., \& Vélez, G. (2014). Enseñanza de la historia reciente y pedagogía de la memoria: emergencias de un debate necesario. Pedagogía y Saberes, (40), 59-70 https://revistas.pedagogica. edu.co/index.php/PYS/article/view/2770

Presidencia de la República de Colombia. (2015, 25 de mayo). Decreto 1038 de 2015. Por el cual se reglamenta la Cátedra de la Paz. https://www.funcionpublica.gov.co/eva/gestornormativo/norma_ pdf.php?i=61735

Ricoeur, P. (2010). Ecrits et conférences, 2. Herméneutique. Du seuil.

Rodríguez, S., \& Sánchez, M. (2009). Problemáticas de la enseñanza de la historia reciente en Colombia: trabajar con la memoria en un país en guerra. Reseñas de Enseñanza de la Historia, (7), 15-66.

Rubio, G. (2010). Memoria y pasado reciente en la experiencia chilena: hacia una pedagogía de la memoria. Universidad de Granada, Facultad de Ciencias de la Educación. http://digibug.ugr.es/ bitstream/10481/4865/1/18654010.pdf

Severino, F. (Org.). (2011). Ética e formação de professores: política, responsabilidade e autoridade em questão. Cortez.

Virkkunen, J. (2006). Dilemmas in building shared transformative. @ctivités, 3(1), 43-66. https://doi. org/10.4000/activites. 1850 